\title{
Frequency bands of strongly nonlinear homogeneous granular systems
}

\author{
Joseph Lydon, ${ }^{1}$ K. R. Jayaprakash, ${ }^{2}$ Duc Ngo, ${ }^{1}$ Yuli Starosvetsky, ${ }^{3}$ Alexander F. Vakakis, ${ }^{2}$ and Chiara Daraio ${ }^{1, *}$ \\ ${ }^{1}$ Graduate Aerospace Laboratories (GALCIT), California Institute of Technology, Pasadena, California 91125, USA \\ ${ }^{2}$ Department of Mechanical Science and Engineering, University of Illinois at Urbana-Champaign, Urbana, Illinois 61801, USA \\ ${ }^{3}$ Department of Mechanical Engineering, Technion-Israel Institute of Technology, Haifa 32000, Israel
}

(Received 5 October 2012; revised manuscript received 25 March 2013; published 19 July 2013)

\begin{abstract}
Recent numerical studies on an infinite number of identical spherical beads in Hertzian contact showed the presence of frequency bands [Jayaprakash, Starosvetsky, Vakakis, Peeters, and Kerschen, Nonlinear Dyn. 63, 359 (2011)]. These bands, denoted here as propagation and attenuation bands (PBs and ABs), are typically present in linear or weakly nonlinear periodic media; however, their counterparts are not intuitive in essentially nonlinear periodic media where there is a complete lack of classical linear acoustics, i.e., in "sonic vacua." Here, we study the effects of PBs and ABs on the forced dynamics of ordered, uncompressed granular systems. Through numerical and experimental techniques, we find that the dynamics of these systems depends critically on the frequency and amplitude of the applied harmonic excitation. For fixed forcing amplitude, at lower frequencies, the oscillations are large in amplitude and governed by strongly nonlinear and nonsmooth dynamics, indicating PB behavior. At higher frequencies the dynamics is weakly nonlinear and smooth, in the form of compressed low-amplitude oscillations, indicating $\mathrm{AB}$ behavior. At the boundary between the $\mathrm{PB}$ and the $\mathrm{AB}$ large-amplitude oscillations due to resonance occur, giving rise to collisions between beads and chaotic dynamics; this renders the forced dynamics sensitive to initial and forcing conditions, and hence unpredictable. Finally, we study asymptotically the near field standing wave dynamics occurring for high frequencies, well inside the AB.
\end{abstract}

DOI: 10.1103/PhysRevE.88.012206

PACS number(s): 45.70.-n, 43.25.+y, 05.45.Yv, 46.40.Cd

\section{INTRODUCTION}

One-dimensional ordered arrays of granular particles exhibit rich dynamical behavior, which has been extensively studied. The dynamics of these media is highly tunable, from strongly nonlinear and nonsmooth in the absence of static precompression, to weakly nonlinear and smooth under large static precompression [1,2]. The nonlinearity in granular media arises from two sources: First, the geometry of the interacting particles leads to a nonlinear Hertzian interaction [3], and second, a tensionless behavior when beads separate. This interaction between neighboring spherical elastic beads may be written as

$$
F=\left\{\begin{array}{ll}
k d^{3 / 2}, & d \geqslant 0 \\
0, & d<0
\end{array} .\right.
$$

Here, $d$ is the overlap displacement between beads, $k$ is the coefficient of the Hertzian interaction, dependent on size and material, and $F$ is the resulting force. Granular crystals have been proposed for a variety of engineering applications; some of these include shock energy trapping [4-6], vibration filtering [7,8], focusing [9], energy harvesting [10], and nonlinear localization [11]. In addition, granular crystals have been shown to support metastable breathers in material systems characterized as "sonic vacuum" [12], and localized oscillations in diatomic crystals [13] and in systems with defects [14,15]. Hence, understanding in detail the mechanisms for controlling energy propagation, attenuation, and localization in granular crystals might lead to new classes of purely passive filtering and protective devices.

\footnotetext{
*Current address: Department of Mechanical and Process Engineering, Swiss Federal Institute of Technology (ETH), Zürich, Switzerland; daraio@ethz.ch
}

This work examines the response of an uncompressed, harmonically driven two-bead system, similar to the one discussed in the theoretical model proposed by Jayaprakash et al. [16]. In contrast to the case of statically precompressed granular crystals, uncompressed granular crystals exhibit strongly nonlinear dynamic behavior. Their response is not linearizable and there is complete absence of classical linear acoustic response. Nesterenko characterized this essentially nonlinear medium as sonic vacuum [1] since the linearized speed of sound (as defined in classical linear acoustics) is zero. Despite the fact that frequency bands are phenomena inherent to linear periodic systems, Jayaprakash et al. [16] demonstrated the existence of similar propagation and attenuation bands in essentially nonlinear uncompressed granular crystals. They predicted that a one-dimensional granular crystal of infinite extent exhibits either propagation or attenuation behavior dependent on both frequency (as is the case in coupled linear periodic oscillators) and amplitude (due to the nonlinearity of the system).

The propagation band $(\mathrm{PB})$ of the system is observed at lower drive frequencies. It is characterized by strongly nonlinear and nonsmooth dynamics, a result of bead separations and collisions. This gives rise to a time-periodic train of traveling pulses, similar to solitary waves analytically predicted and experimentally demonstrated by Nesterenko [1]. At higher frequencies, the attenuation band $(\mathrm{AB})$ is characterized by a region where spatially periodic solutions cannot exist. In this regime, the system exhibits low-amplitude localized oscillations bounded by decaying spatial envelopes, similar to evanescent waves predicted in band gaps of linear media. In this high-frequency regime the chain is dynamically compressed and weakly nonlinear dynamics governs the dynamical response. Jayaprakash et al. [16] predicted that these PBs and ABs exist as well in forced granular media of arbitrary length. We set out to demonstrate this behavior in a harmonically forced system of two beads. 


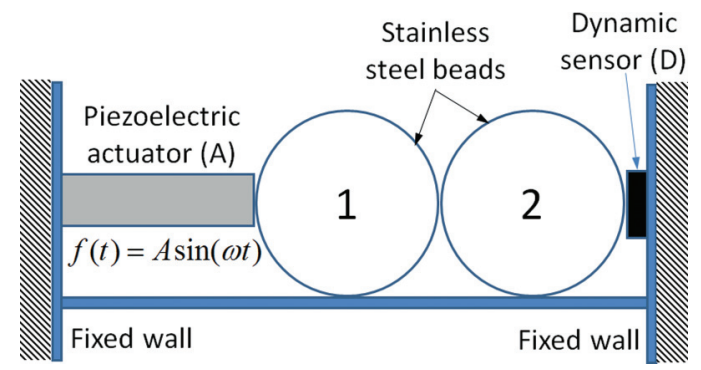

FIG. 1. (Color online) Experimental setup.

\section{EXPERIMENTAL SETUP AND NUMERICAL MODEL}

In our experiments, we consider a system of two beads placed between a dynamic sensor (PCB 208C01) and a piezoelectric actuator (PST 150/5/7 VS10) [17] (Fig. 1). The actuator is used to harmonically excite the first bead. The piezoactive material of the actuator is much stiffer than the actuator cap and acts as a moving wall. We consider the system to be displacement driven; i.e., the transfer of the initial excitation is controlled by Hertzian interactions, as expressed in (2). The dynamic force sensor measures transmitted force and it is used to infer the dynamics and state of the system, i.e., whether the system is driven in a strongly or weakly nonlinear regime. We use two polycarbonate support rods to align the beads, actuator, and dynamic sensor along a common axis. Polycarbonate is chosen to reduce acoustic coupling between the beads and supports. Finally, we adjust the offset bias of the actuator to achieve dynamics at near zero precompression. The dynamics of the experiments is highly dependent upon this initial condition of zero static compression. Reaching and maintaining zero static overlap in longer chains is more difficult and less repeatable. Similar dynamical regimes were observed in chains of three, four, and five beads, but we have chosen to omit the results due to a lack of repeatability in the measurements. We believe such dynamic instabilities to be caused by rotations and misalignment between the particles.

We numerically model the setup shown in Fig. 1 by considering all interactions to be Hertzian and purely elastic. To obtain the coefficient in the Hertzian contact law, we consider the experimental material properties of the beads, actuator, and dynamic sensor. At the boundaries, the particles are in contact with an actuator and a force sensor, both having a planar surface. We model the planar surfaces of the actuator and sensor as spheres with radii tending to infinity. The beads are made of stainless steel 316 and have a Young's modulus $E_{s}=193 \mathrm{GPa}$, Poisson's ratio $v_{s}=0.3$, mass $m=$ $28.84 \mathrm{~g}$, and radius $R=9.525 \mathrm{~mm}$ [18]. The Young's modulus and Poisson ratio for the actuator and dynamic sensor are $E_{a}=193 \mathrm{GPa}, v_{a}=0.3$ and $E_{d}=197 \mathrm{GPa}, v_{d}=0.272$, respectively [18]. The equations of motion for the experimental setup shown in Fig. 1 are given by

$$
\begin{aligned}
m \frac{d^{2} u_{1}}{d t^{2}}= & (4 / 3) E_{1 *} \sqrt{R}\left\{\left[F(t)-u_{1}\right]_{+}^{3 / 2}-\frac{1}{\sqrt{2}}\left(u_{1}-u_{2}\right)_{+}^{3 / 2}\right\}, \\
m \frac{d^{2} u_{2}}{d t^{2}}= & (4 / 3) E_{1 *} \sqrt{R} \frac{1}{\sqrt{2}}\left(u_{1}-u_{2}\right)_{+}^{3 / 2} \\
& -(4 / 3) E_{2 *} \sqrt{R}\left(u_{2}\right)_{+}^{3 / 2},
\end{aligned}
$$

$$
\begin{aligned}
\frac{1}{E_{1 *}} & =\frac{1-v_{A}^{2}}{E_{A}}+\frac{1-v_{S}^{2}}{E_{S}}=2 \frac{1-v_{S}^{2}}{E_{S}}, \\
\frac{1}{E_{2 *}} & =\frac{1-v_{S}^{2}}{E_{S}}+\frac{1-v_{D}^{2}}{E_{D}},
\end{aligned}
$$

where $u_{1}$ and $u_{2}$ are the displacements of beads 1 and 2, respectively, and $E_{1 *}$ and $E_{2 *}$ the effective Young's moduli of the different contact interactions. The subscript " + " is used to capture the tensionless behavior of the particles, signifying that only positive values of the terms inside the parentheses should be considered and set equal to zero otherwise.

To study the forced dynamics of system (2) we impose a harmonic displacement $F(t)=A \sin (\omega t)$ of the left steel wall bounding the system; this wall is assumed to be initially in contact with the first bead. By varying the frequency of the harmonic excitation of the wall we aim to study the dynamics of the granular system, and, specifically, to study the intended transition from a low-frequency $\mathrm{PB}$ to a high-frequency $\mathrm{AB}$ as the frequency increases. In addition, we will discuss interesting complex nonlinear dynamics occurring close to the boundary between the $\mathrm{PB}$ and $\mathrm{AB}$ when large-amplitude oscillations of the bead due to resonance occur. To stabilize the numerical simulations and simulate more accurately the experiment, we add a small amount of artificial viscous damping (equal to $100 \mathrm{~N} \mathrm{~s} / \mathrm{m}$ ). Otherwise, the model does not include any damping or dissipative mechanism which is inherent in the experimental system.

\section{NUMERICAL RESULTS}

In Fig. 2 we present the response of the system for a low excitation frequency of $10 \mathrm{~Hz}$. Note that in all numerical simulations and experiments the excitation amplitude is approximately $0.4 \mu \mathrm{m}$. The force profile in time transmitted to the wall (opposite to the actuator) is plotted in Fig. 2(a). At
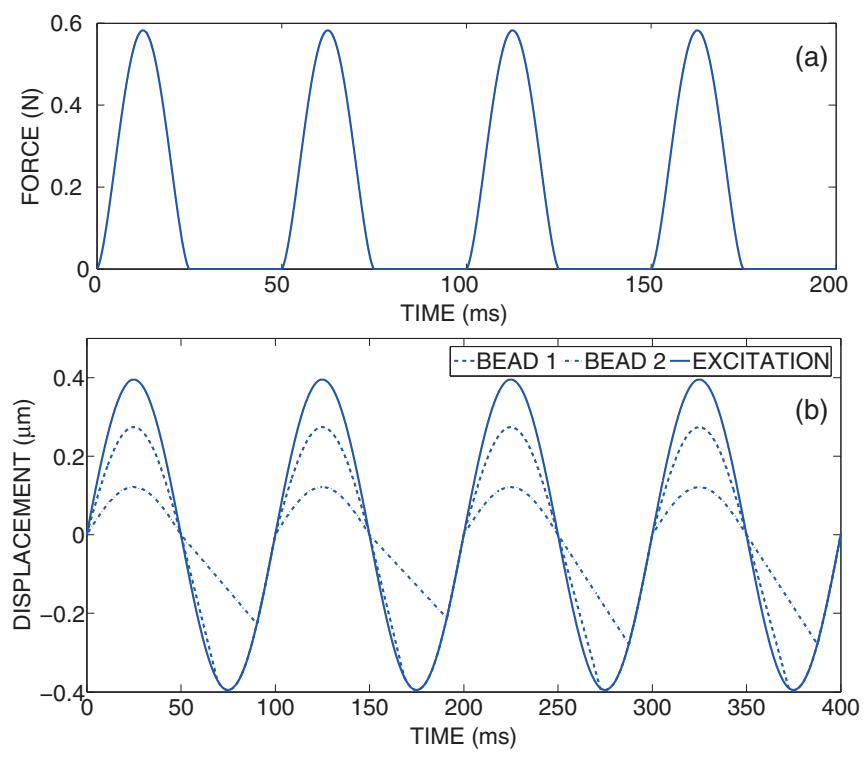

FIG. 2. (Color online) Response of the two-bead system for excitation frequency equaling $f=10 \mathrm{~Hz}$ and amplitude $A=3.951 \times$ $10^{-7} \mathrm{~m}$ : (a) Transmitted force on the boundary (the site of the dynamic sensor), (b) bead displacements. 

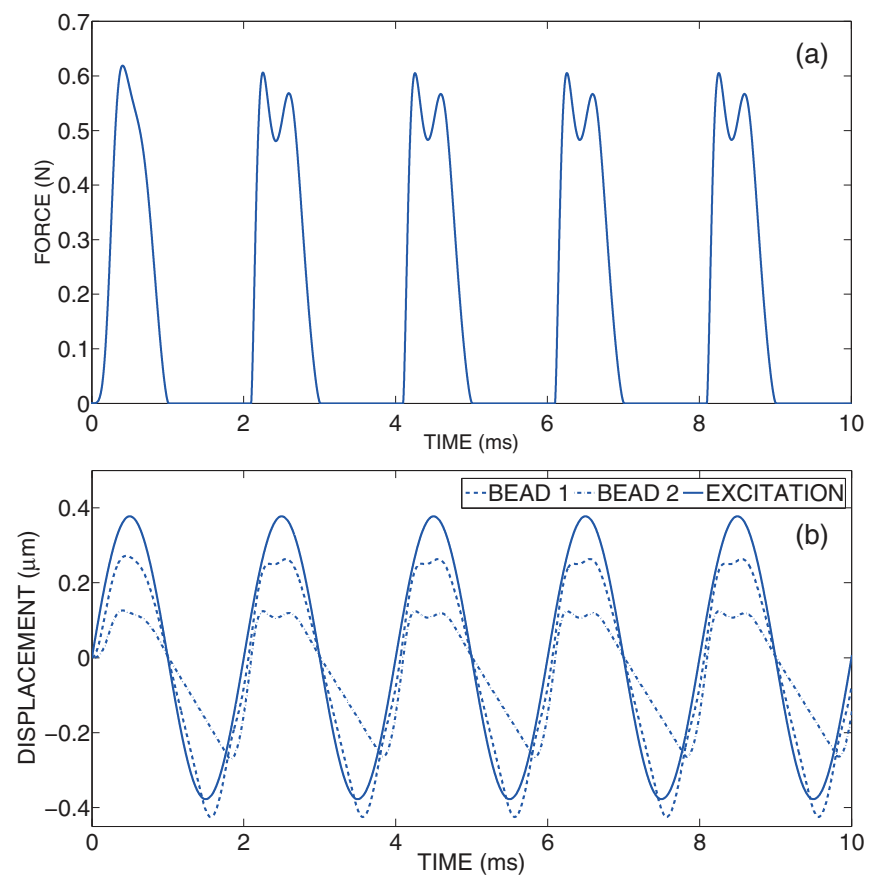

FIG. 3. (Color online) Response of the two-bead system for excitation frequency equaling $f=500 \mathrm{~Hz}$ and amplitude $A=$ $3.775 \times 10^{-7} \mathrm{~m}$ : (a) Transmitted force on the boundary (the site of the dynamic sensor), (b) bead displacements.

this low frequency, the responses of both beads closely follow the excitation [see the temporal evolution of the particles' displacement in Fig. 2(b)]. When the excitation displacement is positive the beads are compressed against the dynamic force sensor, and when the excitation is negative the beads relax and lose contact with the dynamic sensor, resulting in zero applied force. The beads perform strongly nonlinear, nearly in-phase and highly asymmetric oscillations about their equilibrium positions, transmitting a force in the form of a well-separated train of pulses to the sensor. This is indicative of dynamics in a PB of the system. Similar force and displacement responses can be seen at higher excitation frequencies $(500 \mathrm{~Hz}$, Fig. 3 and $1000 \mathrm{~Hz}$, Fig. 4), with the notable difference that at higher frequencies the bead displacements do not follow the motion of the actuator but instead have more complex waveforms.

At higher frequencies, however, we note a qualitatively different behavior in the dynamic response of the particles, since now they execute standing wave oscillations. Indeed, at $3000 \mathrm{~Hz}$ (Fig. 5), the displacement of the beads cannot keep up with the high frequency of the actuator and attain a sustained state of (positive) dynamic compression. Furthermore, at such a compressed state separation between beads is no longer possible. This induces a state of sustained compression [denoted by the nonzero average force in Fig. 5(a)], which changes the dynamic behavior of the system from strongly nonlinear (characteristic of the PB), to weakly nonlinear and spatially localized (characteristics of the $A B$ ). In the $\mathrm{AB}$ the dynamics is weakly nonlinear and, as shown in a later section, can be analyzed by an asymptotic technique. In addition, the amplitude of the oscillation about the mean position of each bead is significantly reduced from bead to
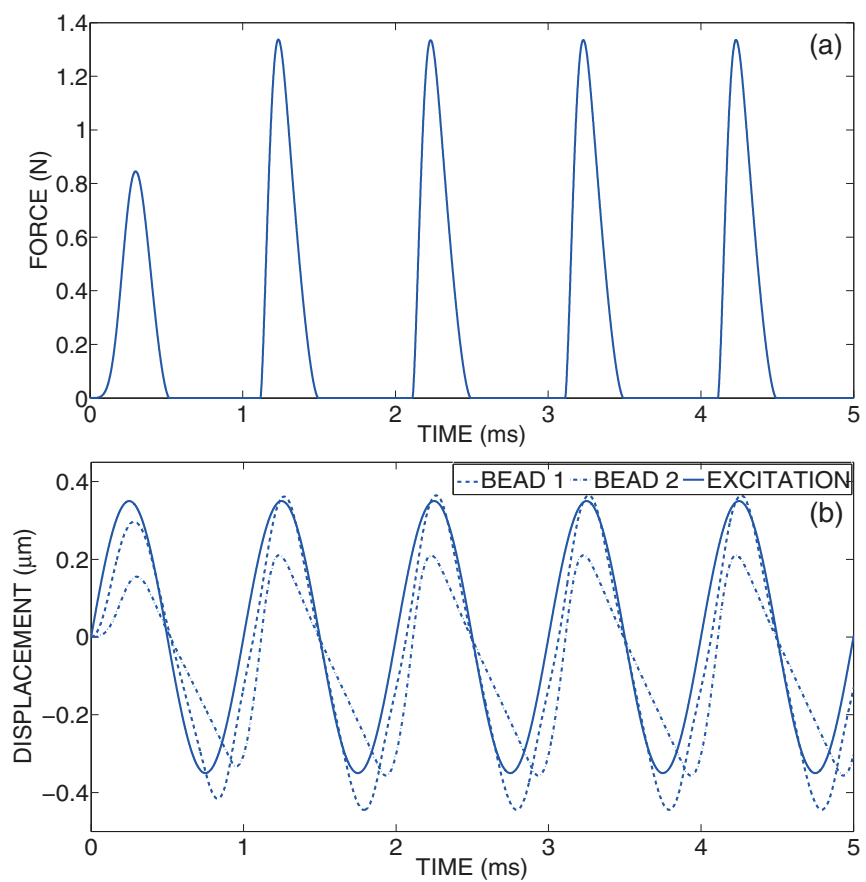

FIG. 4. (Color online) Response of the two-bead system for excitation frequency equaling $f=1000 \mathrm{~Hz}$ and amplitude $A=$ $3.5 \times 10^{-7} \mathrm{~m}$ : (a) Transmitted force on the boundary (the site of the dynamic sensor), (b) bead displacements.

bead [Fig. 5(b)], similar to attenuation in linear media. This change is brought solely by varying the excitation frequency, keeping the excitation amplitude nearly constant.

When increasing the excitation frequency to $5000 \mathrm{~Hz}$ (Fig. 6), the mean force calculated at the wall remains nearly
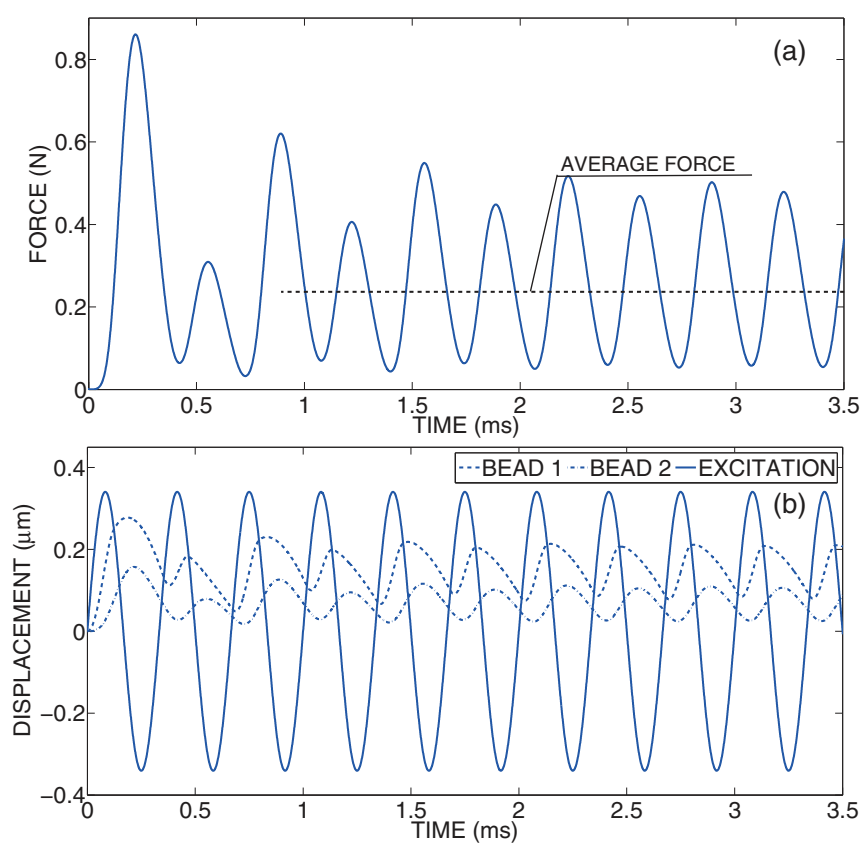

FIG. 5. (Color online) Response of the two-bead system for excitation frequency equaling $f=3000 \mathrm{~Hz}$ and amplitude $A=$ $3.408 \times 10^{-7} \mathrm{~m}$ : (a) Transmitted force on the boundary (the site of the dynamic sensor), (b) bead displacements. 

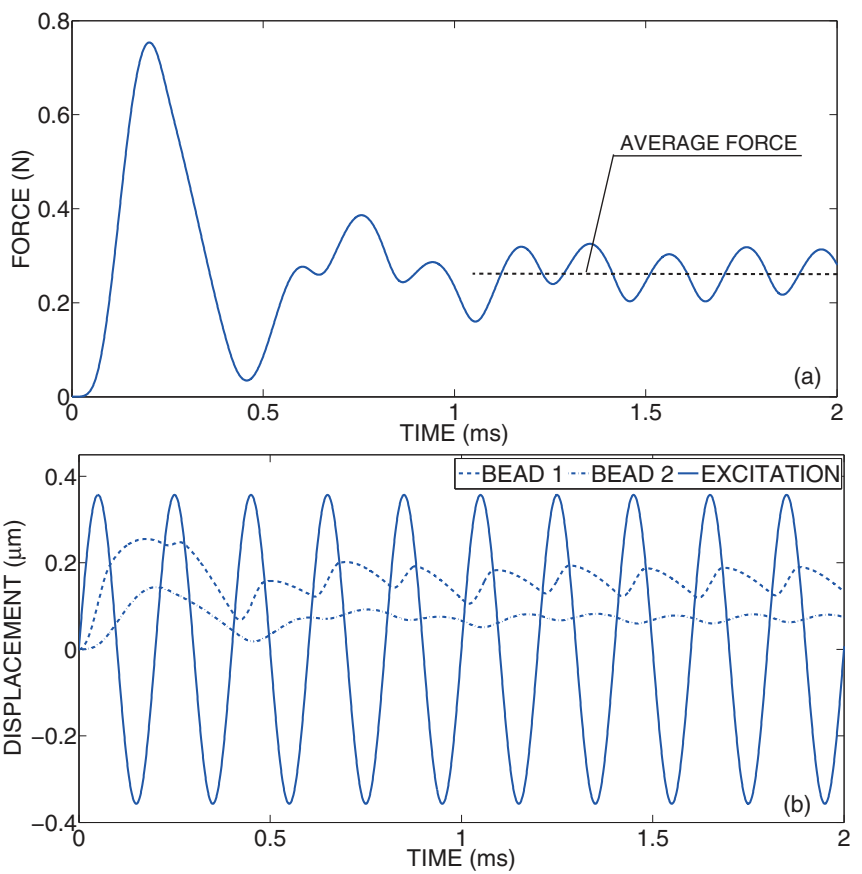

FIG. 6. (Color online) Response of the two-bead system for excitation frequency equaling $f=5000 \mathrm{~Hz}$ and amplitude $A=$ $3.57 \times 10^{-7} \mathrm{~m}$ : (a) Transmitted force on the boundary (the site of the dynamic sensor), (b) bead displacements.

unchanged [Fig. 6(a)], but the oscillation amplitude about the mean value is considerably reduced [Fig. 6(b)]. As the frequency of the excitation increases, the spatial localization of the bead response similarly increases. In conclusion, we note that above $1000 \mathrm{~Hz}$ the system response is in the $\mathrm{AB}$. Figure 7 shows the significant decrease of the power of the higher harmonics of the nonlinear standing wave response when transitioning from the strongly nonlinear dynamics in the lower-frequency PB [Fig. 7(a)] to the weakly nonlinear response in the higher-frequency $\mathrm{AB}$ [Fig. 7(b)]. In addition, the compressed state in the $\mathrm{AB}$ allows us to linearize the system for a more comprehensive analytical study, as described in Sec. V below.

\section{EXPERIMENTAL RESULTS}

We test the experimental setup shown in Fig. 1. We excite the first bead harmonically with amplitudes of approximately $0.4 \mu \mathrm{m}$ (reproduced in the numerical simulations). Small deviations $( \pm 0.05 \mu \mathrm{m})$ from this excitation value occur due to inherent nonlinear behavior of the actuator. We measure the force exerted on the dynamic sensor and show the existence of a high-amplitude strongly nonlinear state at low frequencies (the PB), and a low-amplitude weakly nonlinear state at high frequencies (the $\mathrm{AB}$ ).

In Figs. 8(a) and 8(b), we show the experimental time series of the force measured at the sensor at driving frequencies of 10 and $500 \mathrm{~Hz}$, respectively. These are qualitatively similar to the simulations at the same frequencies. We note a series of transmitted compressive force pulses, similar to the dynamics observed in simulation. At $10 \mathrm{~Hz}$ the width of this pulse is approximately half the period of the drive; however, at $500 \mathrm{~Hz}$
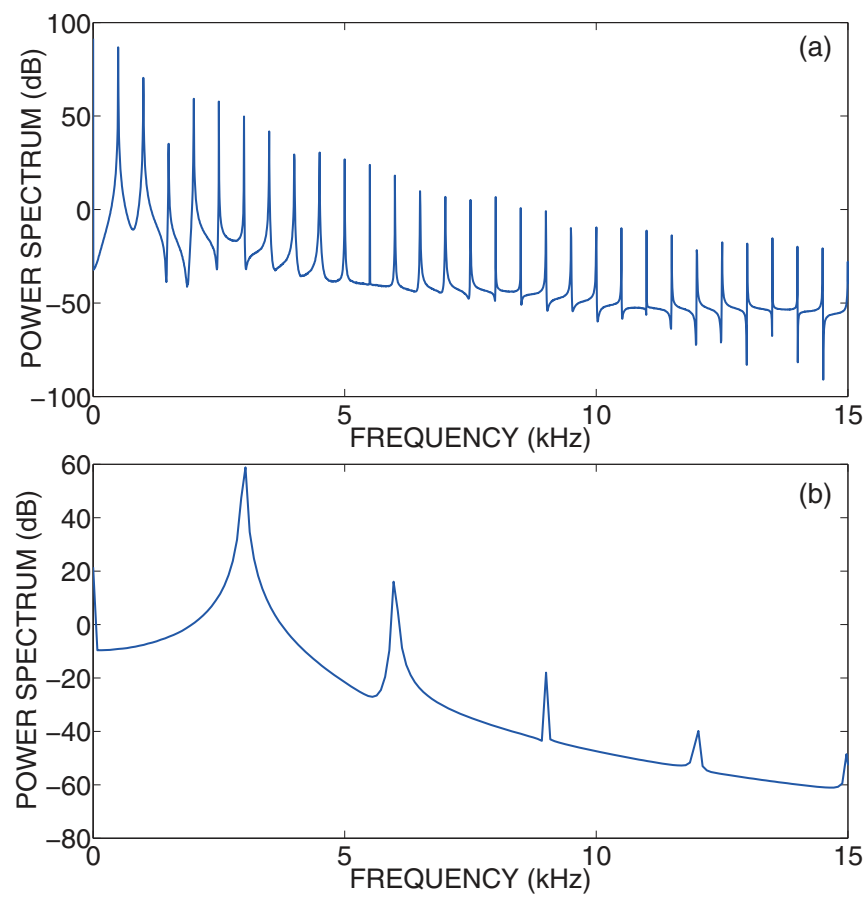

FIG. 7. (Color online) Fourier transform of the transmitted force to the dynamic sensor at forcing frequency equaling (a) $f=500 \mathrm{~Hz}$ and (b) $f=3000 \mathrm{~Hz}$.

this pulse width decreases below half the drive period. This is in agreement the numerical results predicting decreasing pulse width for higher frequencies. The maximum transmitted force is higher at $500 \mathrm{~Hz}$, also in qualitative agreement with the numerical simulations. However, in the numerical simulations
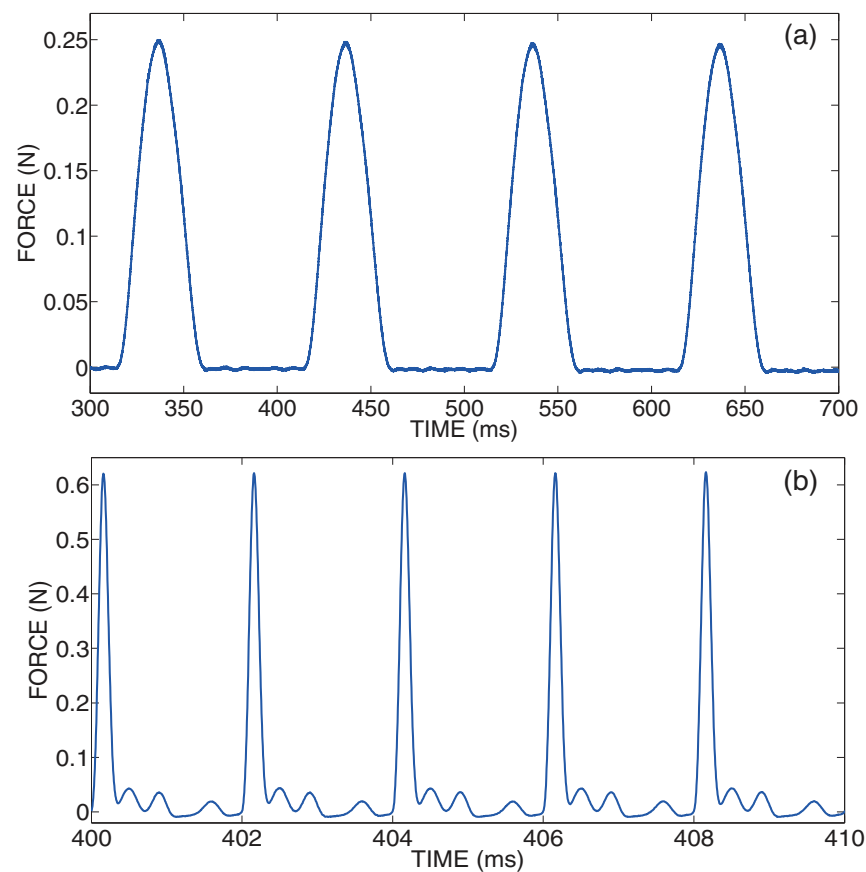

FIG. 8. (Color online) Experimental time series of the force at the dynamic sensor at (a) $f=10 \mathrm{~Hz}$ and $A=3.951 \times 10^{-7} \mathrm{~m}$, and (b) $f=500 \mathrm{~Hz}$ and $A=3.775 \times 10^{-7} \mathrm{~m}$. 
we observed resonance phenomena where the maximum force recorded was much higher at $1000 \mathrm{~Hz}$. The experimental observation of resonances is not included in this paper due to difficulty in experimental repeatability.

A number of experimental uncertainties such as misalignment, surface roughness, and bead rotations become important and difficult to avoid as the displacement amplitudes increase at resonance. In addition, we performed an extensive series of numerical simulations of harmonically forced ordered granular systems close to the boundary between their PB and $\mathrm{AB}$ where nonlinear resonances are excited and the granular media execute large-amplitude oscillations. In these regions, there occur strong collisions between beads, which are well known to give rise to chaotic dynamics [19]. Due to the existence of such chaotic (nonsmooth) motions the forced dynamics of the forced granular systems exhibits sensitive dependence to initial and forcing conditions and become, in essence, unpredictable. This was verified in the experiments where in the resonance region (i.e., for frequencies close to the boundary between the $\mathrm{PB}$ and the $\mathrm{AB}$ ) different experimental runs that were performed under identical forcing and initial conditions yielded completely different results. Close to the boundary between the PB and AB the inherent chaotic dynamics of the harmonically forced system prevents the accurate measurement of the dynamic response, and the resulting chaotic dynamics becomes unpredictable.

By increasing the frequency of the excitation the dynamics becomes again regular and fully predictable (and reproducible). Indeed, a weakly nonlinear regime is found at higher frequencies $[3000 \mathrm{~Hz}$, Fig. 9(a) and $5000 \mathrm{~Hz}$, Fig. 9(b)], with the nonzero mean force indicating a state of sustained compression. The small-amplitude oscillations of the measured force about this mean value indicates weakly nonlinear interactions in the dynamics. Moreover, increasing
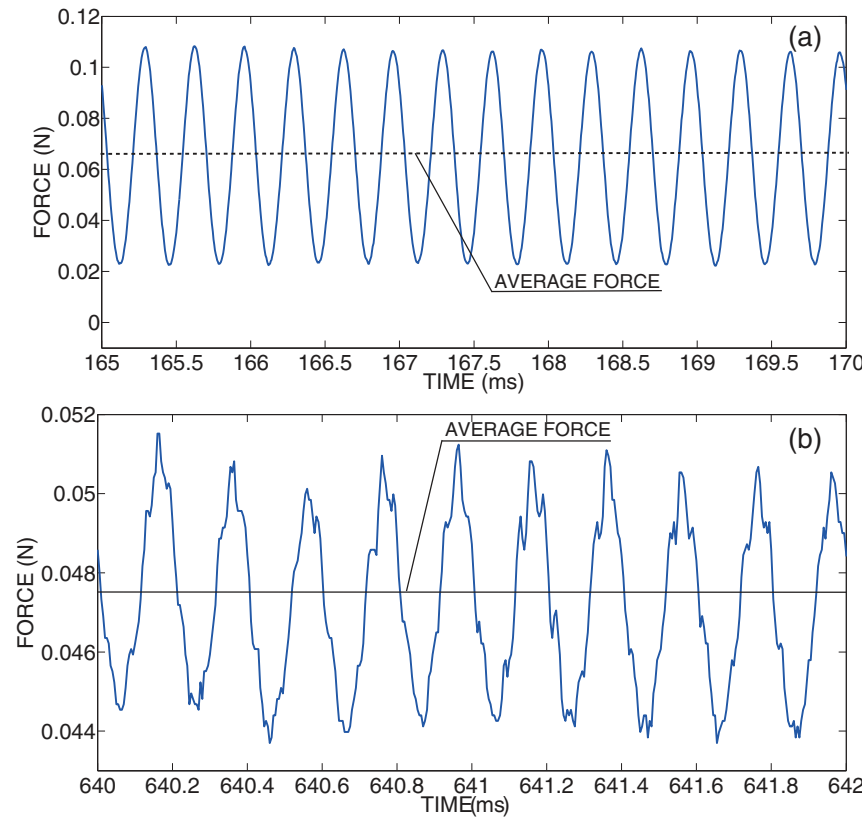

FIG. 9. (Color online) Experimental time series of the force at the dynamic sensor at (a) $f=3000 \mathrm{~Hz}$ and $A=3.408 \times 10^{-7} \mathrm{~m}$, and (b) $f=5000 \mathrm{~Hz}$ and $A=3.57 \times 10^{-7} \mathrm{~m}$.
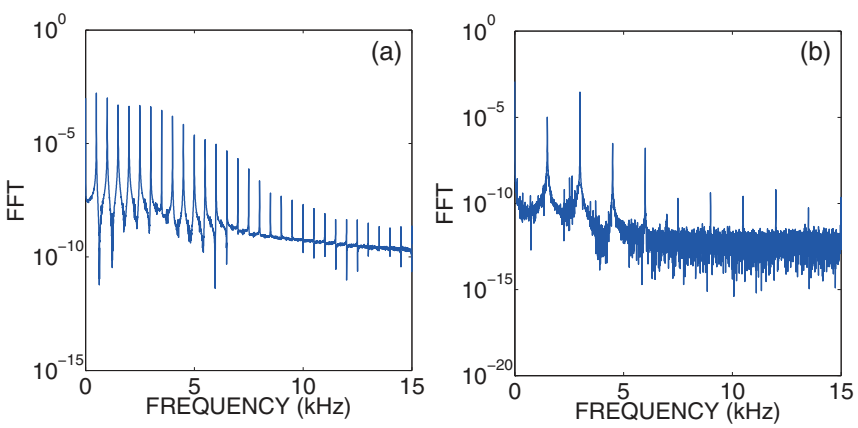

FIG. 10. (Color online) Experimental power spectral densities of the force time series of (a) Fig. 8(b): $f=500 \mathrm{~Hz}$, and (b) Fig. 9(a): $f=3000 \mathrm{~Hz}$.

the frequency decreases the transmitted force amplitude for these oscillations. A comparison of the Fourier spectrum, calculated using a discrete fast Fourier transform, of the dynamics in the PB [Fig. 10(a)] with those in the AB [Fig. 10(b)] underlines that fewer harmonics are excited in the weakly nonlinear phase, in agreement with the simulations. It should be noted that, although the experimental results do not match the numerical results quantitatively, we have good qualitative agreement between the two responses. The mismatch can be attributed to the dry friction, material damping, and other uncertainties present in the experimental setup.

It should be emphasized that the reported experimental results are only for a system of two interacting beads. These experimental results show qualitative agreement with the numerical results for two beads. We believe this validates the modeling and suggests that the numerical results for longer chains hold true. At small initial compressions the experiment demonstrates similar dynamics to that predicted by our analysis for longer chains. We analytically examine the differences in dynamics for chains of arbitrary length below.

\section{ANALYSIS}

In this section, we analytically study the weakly nonlinear dynamics in the AB. The induced permanent compression leads to a linearizable system suitable for analytical techniques. We extend the previous results by considering a homogeneous crystal of $N$ beads. Similar to the setup of Sec. II for two beads, the first bead is harmonically driven and the $N$ th bead is constrained by a fixed wall. All the beads interact with their nearest neighbors through Hertzian interaction law. We incorporate linear viscous damping (coefficient $\lambda$ ) between interacting beads to simulate dissipative effects in the experimental system and to suppress transient dynamics. Here, we account for the damping force only when the beads are in contact and thus incorporate the Heaviside function $(\Theta)$ on the relative displacement of the beads. In our analytical approach we nondimensionalize the equations of motion so that the results are of general applicability. We therefore begin with presenting the equations of motion of the theoretical model of an $N$-bead chain with right end fixed and the 
left end harmonically excited:

$$
\begin{gathered}
m_{1} \frac{d^{2} u_{1}}{d t^{2}}=(4 / 3) E_{1 *} \sqrt{R}\left[F(t)-u_{1}\right]_{+}^{3 / 2}-(4 / 3) E_{*} \sqrt{\frac{R}{2}\left(u_{1}-u_{2}\right)_{+}^{3 / 2}+\lambda\left[\dot{f}(t)-\dot{u}_{1}\right] \Theta\left[f(t)-u_{1}\right]-\lambda\left(\dot{u}_{1}-\dot{u}_{2}\right) \Theta\left[u_{1}-u_{2}\right]} \\
\ldots \\
m_{i} \frac{d^{2} u_{i}}{d t^{2}}=(4 / 3) E_{*} \sqrt{\frac{R}{2}}\left\{\left(u_{i-1}-u_{i}\right)_{+}^{3 / 2}-\left(u_{i}-u_{i+1}\right)_{+}^{3 / 2}\right\}+\lambda\left(\dot{u}_{i-1}-\dot{u}_{i}\right) \Theta\left[u_{i-1}-u_{i}\right]-\lambda\left(\dot{u}_{i}-\dot{u}_{i+1}\right) \Theta\left[u_{i}-u_{i+1}\right] \\
\quad \ldots \\
\left.m_{N} \frac{d^{2} u_{N}}{d t^{2}}=(4 / 3) E_{*} \sqrt{\frac{R}{2}} u_{N-1}-u_{N}\right)_{+}^{3 / 2}-(4 / 3) E_{2 *} \sqrt{R}\left(u_{N}\right)_{+}^{3 / 2}+\lambda\left(\dot{u}_{N-1}-\dot{u}_{N}\right) \Theta\left[u_{N-1}-u_{N}\right]-\lambda\left(\dot{u}_{N i}\right) \Theta\left[u_{N}\right],
\end{gathered}
$$

where $i=2,3, \ldots,(N-1)$ are the bead subscripts and $F(t)=A \sin (\omega t)$ is the harmonic base excitation as defined in Sec. II. The appropriate nondimensionalization leads to the set of normalized equations of motion,

$$
\begin{gathered}
\frac{d^{2} X_{1}}{d \tau^{2}}=\eta\left[\sin (\beta \tau)-X_{1}\right]_{+}^{3 / 2}-\frac{1}{\sqrt{2}}\left(X_{1}-X_{2}\right)_{+}^{3 / 2}+\xi\left[\beta \cos (\beta \tau)-X_{1}^{\prime}\right] \Theta\left[\sin (\beta \tau)-X_{1}\right]-\xi\left(X_{1}^{\prime}-X_{2}^{\prime}\right) \Theta\left[X_{1}-X_{2}\right] \\
\ldots \\
\frac{d^{2} X_{i}}{d \tau^{2}}=\frac{1}{\sqrt{2}}\left\{\left(X_{i-1}-X_{i}\right)_{+}^{3 / 2}-\left(X_{i}-X_{i+1}\right)_{+}^{3 / 2}\right\}+\xi\left(X_{i-1}^{\prime}-X_{i}^{\prime}\right) \Theta\left[X_{i-1}-X_{i}\right]-\xi\left(X_{i}^{\prime}-X_{i+1}^{\prime}\right) \Theta\left[X_{i}-X_{i+1}\right] \\
\quad \ldots \\
\frac{d^{2} X_{N}}{d \tau^{2}}=\frac{1}{\sqrt{2}}\left(X_{N-1}-X_{N}\right)_{+}^{3 / 2}-\alpha\left(X_{N}\right)_{+}^{3 / 2}+\xi\left(X_{N-1}^{\prime}-X_{N}^{\prime}\right) \Theta\left[X_{N-1}-X_{N}\right]-\xi\left(X_{N}^{\prime}\right) \Theta\left[X_{N}\right],
\end{gathered}
$$

where $E_{*}$ is the effective stiffness between the interacting beads, and $X_{i}=u_{i} / A, \tau=\left(4 E_{*} \sqrt{A R} / 3 m\right)^{1 / 2} t \equiv \psi t, \eta=$ $E_{1 *} / E_{*}, \alpha=E_{2 *} / E_{*}, \beta=\omega / \psi, \xi=\lambda / m \psi$ are nondimensional variables relating (3) and (4). Without loss of generality, we consider $\eta=1$ and $\alpha=1$ denoting that the dynamic sensor, actuator, and beads are made of the same material. This does not affect the validity of the resulting dynamics. Recalling the material and experimental data from Sec. II, we obtain $E_{*}=E_{S} /\left[2\left(1-v_{S}^{2}\right)\right], A=3.5 \times 10^{-7} \mathrm{~m}$, and $\omega=8500 \mathrm{~Hz}$; moreover, the nondimensional quantities are computed as $\beta=3.1742, \psi=1.6825 \times 10^{4}$, and $\xi=0.5$.

As a second step, and in view of the fact that a sustained compression is experienced by the beads in the $A B$, we decompose the bead displacements into "static" (permanent constant compression) and "dynamic" (oscillatory) components. For high-frequency excitations in the $\mathrm{AB}$, experiment and simulation indicate that small-amplitude oscillations about a permanent compressed state occur, and this decomposition is in line with this observation. This motivates us to introduce translated coordinates $X_{j}(\tau)=\delta_{j}+x_{j}(\tau)$, where the $j$ th bead's displacement is expressed as a combination of its static, $\delta_{j}>0$, and dynamic, $x_{j}(t)$, components. In simulations we observed that $\delta_{j}>\delta_{j+1}$, i.e., that the permanent compression experienced by each bead decreases as we move away from the actuator and the standing wave oscillation in the $\mathrm{AB}$ becomes spatially localized (Fig. 11). The axes in Figs. 11-15 denote the nondimensional units derived above. From the previously shown results it can be deduced that no separation occurs between beads once the dynamics enters the $A B$, i.e., the dynamics is smooth between interacting beads. Therefore the subscript + can be eliminated from the equations of motion (4), which greatly facilitates the asymptotic analysis. The only exception is the contact between the actuator and first bead.

When the dynamics is well inside the $\mathrm{AB}$ it holds that $\left|\sin (\beta \tau)-\delta_{1}\right| \gg x_{1}(\tau)$, except in the close neighborhood of points "a" and "b" as shown in Fig. 12(a). Thus, it can be assumed that $\left[\sin (\beta \tau)-\delta_{1}-x_{1}\right]>0$ in the region between points $\mathrm{a}$ and $\mathrm{b}$ where the actuator displacement exceeds the permanent compression of the first bead $\delta_{1}$, and $\left[\sin (\beta \tau)-\delta_{1}-x_{1}\right]<0$ when the actuator displacement is less than $\delta_{1}$. Figure 12(b) shows a detailed view of the region between points $a$ and $b$. It follows that we can modify (4) by decomposing the responses in terms of static and dynamic components and incorporating the observations mentioned above. This leads to the following modified equations:

$$
\begin{aligned}
& \frac{d^{2} x_{1}}{d \tau^{2}}= \eta\left[\sin (\beta \tau)-\delta_{1}\right]_{+}^{3 / 2}-\frac{3}{2} \eta\left[\sin (\beta \tau)-\delta_{1}\right]_{+}^{1 / 2} x_{1}-\frac{1}{\sqrt{2}}\left(\delta_{1}-\delta_{2}\right)^{3 / 2}-\frac{3}{2 \sqrt{2}}\left(\delta_{1}-\delta_{2}\right)^{1 / 2}\left(x_{1}-x_{2}\right) \\
&+\xi\left[\beta \cos (\beta \tau)-x_{1}^{\prime}\right] H\left[\sin (\beta \tau)-\delta_{1}\right]-\xi\left(x_{1}^{\prime}-x_{2}^{\prime}\right)+O\left(x_{1}^{p} x_{2}^{q}\right) \\
& \cdots \\
& \frac{d^{2} x_{i}}{d \tau^{2}}= \frac{1}{\sqrt{2}}\left\{\left(\delta_{i-1}-\delta_{i}\right)^{3 / 2}+\frac{3}{2}\left(\delta_{i-1}-\delta_{i}\right)^{1 / 2}\left(x_{i-1}-x_{i}\right)-\left(\delta_{i}-\delta_{i+1}\right)^{3 / 2}-\frac{3}{2}\left(\delta_{i}-\delta_{i+1}\right)^{1 / 2}\left(x_{i}-x_{i+1}\right)\right\} \\
&+\xi\left(x_{i-1}^{\prime}-2 x_{i}^{\prime}+x_{i+1}^{\prime}\right)+O\left(x_{i-1}^{p} x_{i}^{q}\right)+O\left(x_{i}^{r} x_{i+1}^{s}\right)
\end{aligned}
$$




$$
\begin{aligned}
\frac{d^{2} x_{N}}{d \tau^{2}}= & \frac{1}{\sqrt{2}}\left(\delta_{N-1}-\delta_{N}\right)^{3 / 2}+\frac{3}{2 \sqrt{2}}\left(\delta_{N-1}-\delta_{N}\right)^{1 / 2}\left(x_{N-1}-x_{N}\right)-\alpha\left(\delta_{N}\right)^{3 / 2}-\frac{3}{2} \alpha\left(\delta_{N}\right)^{1 / 2} x_{N} \\
& +\xi\left(x_{N-1}^{\prime}-2 x_{N}^{\prime}\right)+O\left(x_{N-1}^{p} x_{N}^{q}\right)+O\left(x_{N}^{z}\right), \quad i=2,3, \ldots,(N-1), p+q \geqslant 2, r+s \geqslant 2, z \geqslant 2 .
\end{aligned}
$$

As in the previous discussion, the only nonsmooth component is in the first equation of (5), modeling the separation of the actuator and first bead.

We observe that there are two terms on the right-hand side of (5) resulting from the interaction between beads; namely static components dependent only on $\delta_{j}$, and dynamic components involving $x_{j}(\tau)$. We account for the nonsmooth terms in (5) by expanding the harmonic excitation term $\left(\left[\sin (\beta \tau)-\delta_{1}\right]_{+}^{3 / 2}\right)$ in Fourier series to obtain static and dynamic components as follows:

$\left[\sin (\beta \tau)-\delta_{1}\right]_{+}^{3 / 2}=a_{0}+\sum_{n=1}^{\infty} a_{n} \cos (n \beta \tau)+\sum_{n=1}^{\infty} b_{n} \sin (n \beta \tau)$

with the coefficients defined as

$$
\begin{aligned}
& a_{0}=\frac{\beta}{2 \pi} \int_{-\pi / \beta}^{\pi / \beta}\left[\sin (\beta \tau)-\delta_{1}\right]_{+}^{3 / 2} d \tau, \\
& a_{n}=\frac{\beta}{\pi} \int_{-\pi / \beta}^{\pi / \beta}\left[\sin (\beta \tau)-\delta_{1}\right]_{+}^{3 / 2} \cos (n \beta \tau) d \tau, \\
& b_{n}=\frac{\beta}{\pi} \int_{-\pi / \beta}^{\pi / \beta}\left[\sin (\beta \tau)-\delta_{1}\right]_{+}^{3 / 2} \sin (n \beta \tau) d \tau .
\end{aligned}
$$

Now, by balancing the static components in all (smooth) equations (5), we obtain the following recursive relation that can be used for computing the permanent compression between beads:

$$
\begin{aligned}
\left(\delta_{j-1}-\delta_{j}\right)^{3 / 2}-\left(\delta_{j}-\delta_{j+1}\right)^{3 / 2} & =0 \\
\cdots & \\
\frac{1}{\sqrt{2}}\left(\delta_{N-1}-\delta_{N}\right)^{3 / 2}-\alpha\left(\delta_{N}\right)^{3 / 2} & =0
\end{aligned}
$$

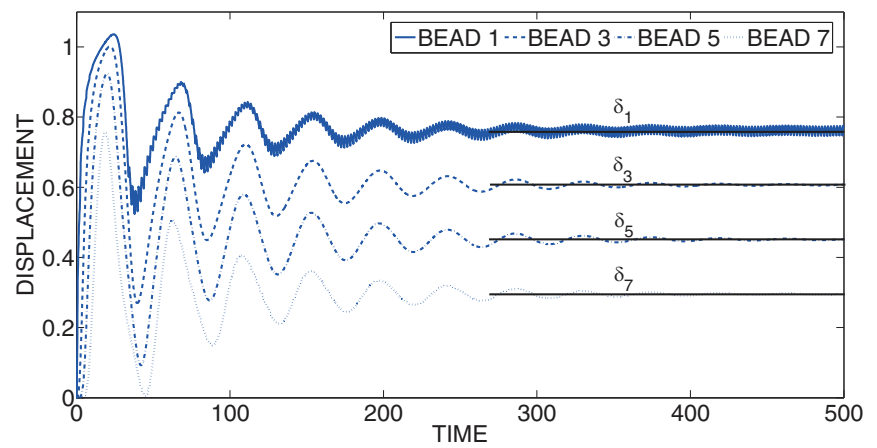

FIG. 11. (Color online) The displacement response of first, third, fifth, and seventh beads of a ten-bead homogeneous chain under harmonic excitation with frequency in the attenuation zone. All units are nondimensional. where $j=2,3, \ldots,(N-1)$. A trivial algebraic manipulation yields

$$
\begin{aligned}
\delta_{j-1} & =2 \delta_{j}-\delta_{j+1}, \\
& \cdots \\
\delta_{N-1} & =\delta_{N}(1+\gamma), \\
\delta_{i} & =\frac{\delta_{i-1}[1+(N-i) \gamma]}{[1+(N-i+1) \gamma]}, \quad 1<i \leqslant N,
\end{aligned}
$$

where $\gamma=2^{1 / 3} \alpha^{2 / 3}$. The only unknown in the above set of equations is the permanent compression of the first bead, $\delta_{1}$. Once this is evaluated the compression of all the other beads can be expressed in terms of $\delta_{1}$. The compression $\delta_{1}$ can be obtained by balancing the static force components in the first equation of (5), i.e., balancing the constant term from the Fourier series (6) with the constant force interaction between the first and the second beads. Then, we obtain an implicit relation of the form

$$
\frac{\eta \beta}{2 \pi} \int_{-\pi / \beta}^{\pi / \beta}\left[\sin (\beta \tau)-\delta_{1}\right]_{+}^{3 / 2} d \tau-\frac{1}{\sqrt{2}}\left(\delta_{1}-\delta_{2}\right)^{3 / 2}=0 .
$$

Substituting for $\delta_{2}$ in terms of $\delta_{1}$ and rescaling time $\tilde{\tau}=\beta \tau$, we derive the final form for the equation governing the permanent
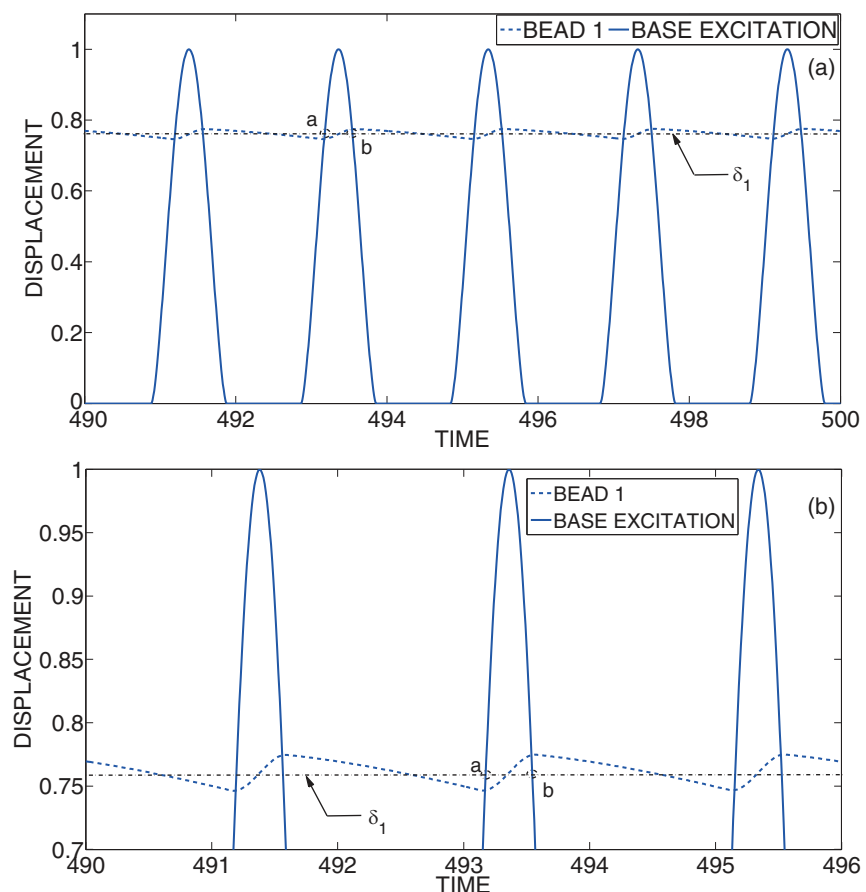

FIG. 12. (Color online) The displacement response of the tenbead homogeneous chain under harmonic excitation with frequency in the attenuation zone: (a) Response of the first bead superimposed to the excitation, and (b) detail of (a). All units are nondimensional. 


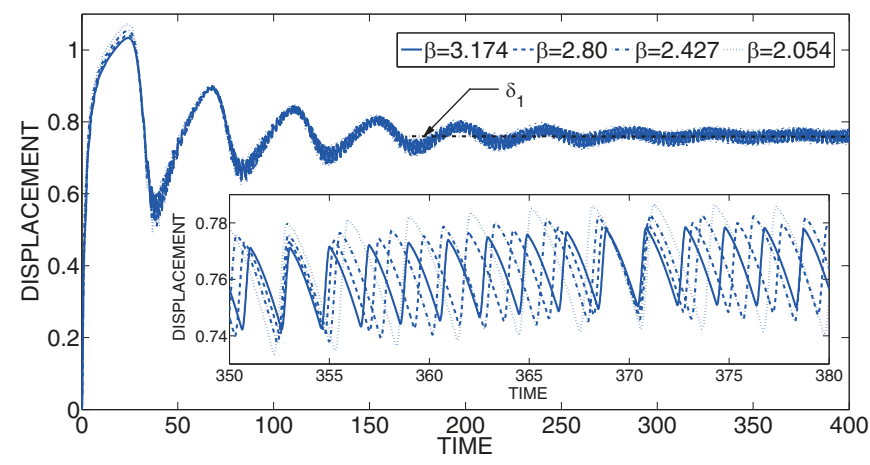

FIG. 13. (Color online) The displacement response of the first bead of a ten-bead homogeneous chain with varying normalized excitation frequency and fixed normalized excitation amplitude equaling unity (dynamics deep inside the attenuation zone). All units are nondimensional.

compression of the first bead,

$$
\left\{\frac{\eta}{\pi} \int_{\sin ^{-1}\left(\delta_{1}\right)}^{\pi / 2}\left[\sin (\tilde{\tau})-\delta_{1}\right]^{3 / 2} d \tilde{\tau}\right\}^{2 / 3}=\delta_{1}\left[\frac{1}{1+(N-1) \gamma}\right]
$$

where the + sign is removed from (8b) since $\left[\sin (\tilde{\tau})-\delta_{1}\right]^{3 / 2} \geqslant 0$ within the limits of integration.

The above equation is evaluated numerically to obtain $\delta_{1}$. This analysis predicts that the static compression $\delta_{1}$ of the first bead (and therefore of any other bead) is independent of the excitation frequency when the dynamics is well inside the $A B$ (i.e., for relatively high frequencies). This is verified through numerical simulations (Fig. 13), where we depict the response of the first bead for a homogeneous chain with $N=10$ at various excitation frequencies $\beta$ inside the $\mathrm{AB}$. The static component of the response is independent of the excitation frequency. This analysis assumes weakly nonlinear behavior, and these predictions are not valid at lower frequencies as the dynamics makes the transition from the PB to the AB.

The static overlap of each bead in the granular chain is evaluated using (8) and (9) and is compared to the results derived from numerical simulation for a 20-bead crystal, $N=20$ (Fig. 14). The numerical simulations show good

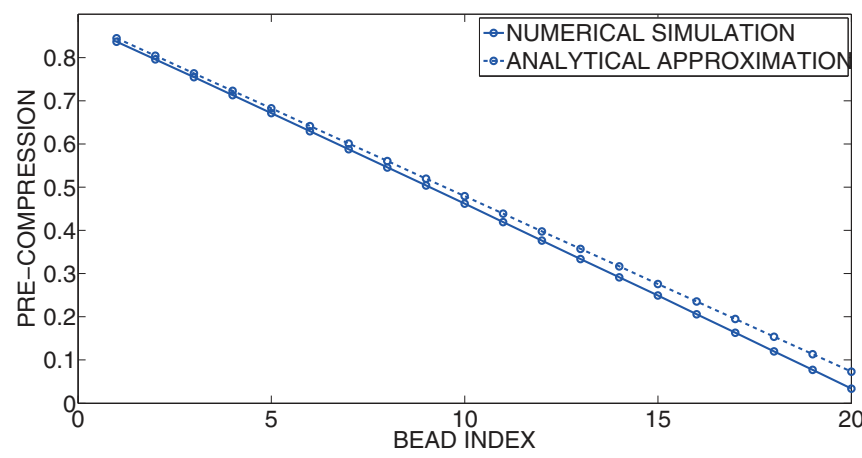

FIG. 14. (Color online) Spatial variation of permanent precompression $\left(\delta_{i}\right)$ in a 20-bead chain when the dynamics is deep in the attenuation zone. All units are nondimensional.

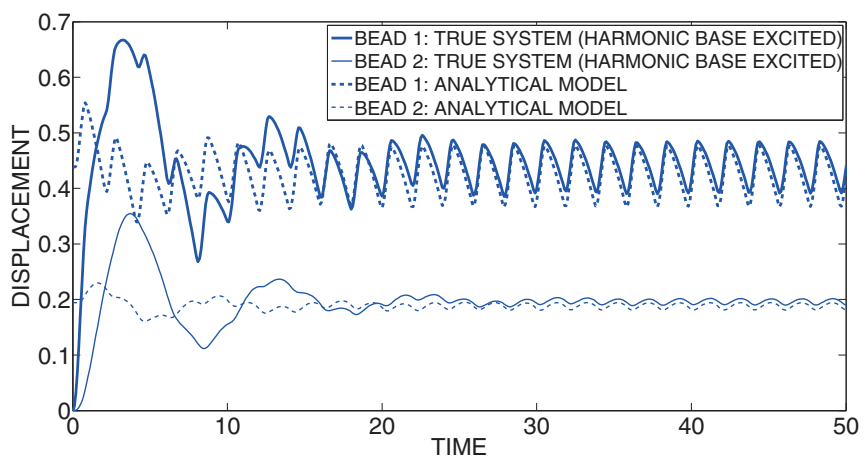

FIG. 15. (Color online) Correspondence between analytical and numerical response of the two-bead system when the dynamics is in the attenuation zone. All units are nondimensional.

correspondence with the analytical estimates and confirm that the spatial variation of the static overlap is nearly linear. As the length of the crystal is increased the static component of the first bead's displacement reaches unity asymptotically, whereas the static offset of the last bead approaches zero. Hence, the resulting standing wave oscillations executed by the beads become spatially localized well inside the $\mathrm{AB}$ of the harmonically forced system. In essence, for a sufficiently large number of beads, the chain detaches from the exciter and the energy input to the chain approaches zero asymptotically.

Finally, we obtain analytic estimates of the individual oscillatory responses of the beads by considering the dynamic components of the bead displacements in (5). We arrive at reduced dynamic equations for each $x_{j}(\tau)$ by removing the static components of (5). These reduced equations depend on the dynamic components of the Fourier series expansion (6). We then find analytic approximations for the oscillatory components of the bead responses for an $N$ degree of freedom linear damped oscillator system with periodically varying forcing frequency. The presence of damping terms leads to steady state periodic responses. Due to the presence of damping, the amplitudes of the dynamic components of the bead responses (i.e., the oscillations about the beads' static offsets) decrease with increasing frequency. For a particular fixed frequency these amplitudes decrease away from the site of the actuator, i.e., $x_{j}(\tau)>x_{j+1}(\tau)$, confirming the attenuating nature of the dynamics, as described above.

The agreement between numerical and analytical response for the forced two-bead system is presented in Fig. 15. The analytical responses closely match the numerical ones, and both $x_{1}(\tau)>x_{2}(\tau)$ and $\delta_{1}>\delta_{2}$. Although the transient dynamics is not captured by our analytic study, the steady state dynamics shows good correspondence between analytical prediction and numerical simulation.

\section{CONCLUDING REMARKS}

This paper explores the presence of frequency bands in harmonically forced essentially nonlinear granular crystals. For fixed amplitude of excitation, the low-frequency dynamics is found to be strongly nonlinear, involving bead separations and collisions, and resulting in periodic trains of traveling 
solitary pulses. This represents the dynamics in a propagation band (PB) of the system. As we increase the drive frequency, the system enters into a state of permanent compression which results in weakly nonlinear and smooth dynamics. In this regime, the response is localized close to the actuator's excitation and rapidly decays away from it. In contrast to the propagatory dynamics realized in the PB, the higherfrequency dynamics is in the form of spatially decaying (and, hence, spatially localized) standing wave oscillations. This represents the attenuation band $(\mathrm{AB})$ of the system. Between these two regimes, nonlinear resonance phenomena occur, where the dynamics becomes chaotic due to strong collisions between beads; the dynamics exhibits sensitive dependence on initial and forcing conditions and becomes unpredictable. This regime was not considered in this work.

Finally, when the dynamics is realized in the $A B$, we employed an asymptotic technique based on static and dynamic partitions of the bead responses, and analytically deduced that the sustained state of compression realized in the granular crystal becomes independent of the excitation frequency. However, an increase in the size of the granular crystal does increase the permanent compression which reduces the amount of energy transferred to the crystal. These results can contribute to designing of granular-based acoustic metamaterials as acoustic filters and attenuators of externally applied periodic or transient disturbances.

\section{ACKNOWLEDGMENTS}

This work was funded in part by MURI Grant No. US ARO W911NF-09-1-0436 and by the US National Science Foundation, NSF CMMI Grant No. 844540. We acknowledge Dr. Nicholas Boechler for assisting with the initial setup of the experiment.
[1] V. Nesterenko, Dynamics of Heterogeneous Materials (Springer, New York, 2001).

[2] C. Daraio, V. F. Nesterenko, E. B. Herbold, and S. Jin, Phys. Rev. E 73, 026610 (2006).

[3] K. L. Johnson, Contact Mechanics (Cambridge University Press, Cambridge, 1987)

[4] C. Daraio, V. F. Nesterenko, E. B. Herbold, and S. Jin, Phys. Rev. Lett. 96, 058002 (2006).

[5] F. Fraternali, M. A. Porter, and C. Daraio, Mech. Adv. Mater. Struct. 17, 1 (2010).

[6] J. Hong, Phys. Rev. Lett. 94, 108001 (2005).

[7] N. Boechler, J. Yang, G. Theocharis, P. G. Kevrekidis, and C. Daraio, J. Appl. Phys. 109, 074906 (2011).

[8] E. B. Herbold, J. Kim, V. F. Nesterenko, S. Wang, and C. Daraio, Acta Mech. 205, 85 (2009).

[9] A. Spadoni and C. Daraio, Proc. Natl. Acad. Sci. USA 107, 7230 (2010).

[10] R. P. Simion and S. Sen, Proc. Inst. Mech. Eng., Part I: J. Syst. Control Eng. 225, 522 (2011).
[11] G. Theocharis, N. Boechler, P. G. Kevrekidis, S. Job, M. A. Porter, and C. Daraio, Phys. Rev. E 82, 056604 (2010).

[12] S. Sen and T. R. Krishna Mohan, Phys. Rev. E 79, 036603 (2009).

[13] A.-C. Hladky-Hennion, G. Allan, and M. de Billy, J. Appl. Phys. 98, 054909 (2005).

[14] S. Job, F. Santibanez, F. Tapia, and F. Melo, Phys. Rev. E 80, 025602 (2009).

[15] G. Theocharis, M. Kavousanakis, P. G. Kevrekidis, C. Daraio, M. A. Porter, and I. G. Kevrekidis, Phys. Rev. E 80, 066601 (2009).

[16] K. Jayaprakash, Y. Starosvetsky, A. F. Vakakis, M. Peeters, and G. Kerschen, Nonlinear Dyn. 63, 359 (2011).

[17] Piezomechanik (2010), http://www.piezomechanik.com/en/ products/.

[18] Metals Handbook, 10th ed. (ASM International, Materials Park, $\mathrm{OH}, 1990)$.

[19] P. J. Holmes, J. Sound Vib. 84, 173 (1982). 